CASE III. A gentleman, some time since under my treatment for stricture, informed me that he could not eat figs without experiencing a most unpleasant formication of the palate and fauces; and that the fine dust from split peas produced the same sensation, accompanied by a running at the nose. The father of this gentleman suffers from hay-fever at certain seasons.

CASE IV. Mr. P., himself a gentleman of a peculiarly nervous temperainent, states that his father cannot endure the sensation produced by handling a russet apple. He also communicated to me

CASE Y-that of Mr. T., who cannot remain in a room in which there is a coolied hare, on account of the peculiar effect produced on his system.

CASE v. Miss - after eating egg, suffers from swelling of the tongue and throat, accompanied by "alarming illness".

CASE VII. Miss —. In this case, somewhat similar effects follow the taking of honey of any kind, and especially honeycomb, into the stomach; viz., swelling of the tongue, frothing of the mouth, and blueness of the fingers.

CASE VIIr. The following is an extract from a note received by me from a lady, who says:-" I had on three mustard plaisters-one on the throat, one on the back of the neck, and another under the left shoulder. They remained on half an hour. Cotton wool was applied on their removal. About thirty hours afterwards, a painful stinging sensation commenced in the back of the neck, followed by violent twitching of the muscles of the face, arms, and legs, which continued in regular succession through the whole night. It yielded, after about twelve hours, to hot fomentations of poppy-heads applied to the back of the neck." It cannot be ascertained that any medicine containing strychnia was taken.

CASE 1x. A gentleman, a member of the medical profession, with whom 1 am well acquainted, suffers from nettle-rash after eating veal. Veal has the reputation of being particularly indigestible. The above instarice of the production of urticaria from its use is, doubtless, not an uncommon one.

CASE $x$. I have been informed of a lady who cannot remain in a room in which there is a cat. Although the cat may be concealed, the lady's peculiar sensations immediately declare to her the presence of the animal.

Case xi. A patient under my care, since dead of cancer, was invariably thrown into a state of nervous excitement by the exhibition of the compound infusion of orange-peel.

CASE XII. A personal friend of my own suffered from erythema nodosum after eating shrimps, although these were perfectly fresh. I believe shell-fish generally is particularly liable to excite unpleasant consequences.

It would be useful, I venture to suggest, if other members of the Association would contribute any instances of idiosyncratic poisoning they may know; since, from a multiplicity of exceptions, some general rule may be educed.

8, Stratford Place, W., June 1859.

\section{ON THE INFLUENCE OF VITALITY UPON THE EXCRETIONS.}

By T. Inנin, M.D., Liverpool.

Ix a recent number, I ventured to call the attention of the profession to the influence which a debilitated condition of the system had upon secretion generally: I wish now to call attention to the influence the same cause has upon the secreted matters themselves; or, as they are sometimes designated, the excretions.

All of us are more or less familiar with the fact that the excretions do not decompose, as a general rule, while they are in the body. The fxces, retained though they be sometirnes for days, and in some rare cases for weeks and months, in the colon or the rectum, do not decompose there, as they do when they are expelled from the body; and this observation holds good, even though the bowels are distended with flatus, and the ficcal matter is in contact with a gas differing in no degree from common atmospheric air. The urine, when retained in the bladder, becomes denser, and undergoes some change; but it does not decompose in the same manner in the interior of the body as it does when expelled therefrom, and kept in a close well stoppered bottle. We account for this by saying that the excreta have a certain vital power as long as they remain in the body, which enables them to resist the ordinary laws governing the inorganic worid.
But we know from experience that the vital power does not immediately leave a limb after it has been amputated; and it becomes, therefore, a question whether vitality may not also remain in excretions for some time after their expulsion from the body. In other words, do the excretions lose their vitality as soon as they are expelled? or is there a period during which they successfully resist chemical laws? And, if such period exist, is it definite for all? Do the excretions of some decompose, cateris paribus, sooner than those of others? and, if so, can we associate that phenomenon in any way with the condition of the individual at the time?

My attention was first called to this subject in the following manner. When M. Ledoyen came to Liverpool, about twelve years ago, to demonstrate the deodorising power of his "disinfectant" fluid, with other experiments, the following was made. The alvine dejections of a certain number of patients, ill with fever and various other diseases, were all placed side by side, to the number of thirty or more, in a small room attached to the pauper hospital. They remained all night in the chamber, and the next day $M$. Ledoyen commenced operations. After demonstrating the general advantages of his compound, he proceeded to sprinkle a few drops of his diluted mixture into each utensil. The amount used was the same in each case but the appearance produced varied immensely ; and, according as the chemical change was excessive or otherwise, he judged of the condition of the individuals who had passed the "motions". "This patient", he would say, " is not very bad; that one is seriously ill ; this one is dying; this one is nearly dead ;" etc. As his observations were correct, he was asked how he judged of the danger the patient was in? His reply was, "that he had found, in the course of his experiments, that freces decomposed rapidly or otherwise, according to the debility of the individual passing them."

For a long period, the principle here enunciated seemed to belong to the class of interesting but useless facts. More re cent observations have, however, shown that it may be turned to good practicai account.

If any one will diligently consult the napkins used by infants, he will find that, during the time the motions are of $\mathbf{a}$ good healthy yellow colour, they have a peculiar odour, which they retain for twelve hours at least; but if, from any cause$e . g$., debility in the nurse, or inappropriateness of the foodthe child loses its healthy condition, the motions not only change in colour and consistence, but in smell, and decompose in a very short time after being passed. Where there is diarrhœa and excessive depression of the vital powers, the motions are often found to be decomposed in a few minutes. We may notice, too, that a similar result is met with at the same time in the other secretions of the child; and that the urine decomposes quickly, and the breath is foul or sickly.

But it is not in children alone that this change may be detected: it is equally evident in adults. If, for example, the doctor is called to attend a case of diarrhœa, where there is alway's more or less debility present, he may consider it neces sary to inspect all the alvine discharges that take place. His visits are at intervals of twelve hours only; and he has on each occasion placed before him perhaps as many as six motions in different utensils. He is probably struck with the different odour exhaled from the various specimens, and notices a difference in the colour; but a few words from the nurse soon explain the mystery. The dark brown stinking ones are those passed the longest period ago; the healthy looking and smelling ones are those passed only a short time before the doctor's visit.

Simple though this fact seems to be, it is one which is not universally acknowledged and acted on; I have known " motions" which have simply become decomposed taken for "foul secretions", and the patient dosed with mercurials, under the inupression that they would improve the condition of the boweis. The result has been what might liave been anticipated; the patient has got weaker, and the bowels no better. The following case came under my notice some time ago. It is valuable as illustrating the danger resulting from inatention to these points.

An elderly gentleman was under treatment for indigestion. $\mathrm{He}$ was improving upon a tonic plan of treatment, when he was induced by his friends to have " a second opinion". When the physician called, he was shown a motion which had been passed twelve hours before. It had undergone decomposition, and was pronounced to be extremely "vitiated"; and, with the intention of improving the secretion, a mercurial alterative was prescribed. This acted freely; and when the visit was made the next day, the motions were all inspected, and as that 
passed the last seemed to be the most healthy, the natural conclusion was that the medicine had done good. It was therefore persevered with; on the next occasion, and for some time subsequently, only the last motion passed was inspected, and, as it had not had time to be decomposed, it was thought to be healthy, and the patient was supposed to be "better", as his secretions were no longer "vitiated". But, notwithstanding this opinion, it was clear to the first attendant that the man was getting more feeble and debilitated day by day. If the medicine did improve the secretions, it certainly impaired the strength. It was then doubted whether the first inference was correct; and, to decide this, the nurse was directed to save all the "motions", and arrange them in the order in which they were passed. When they were inspected, the same order of things was noted as at the first examination; but the older ones appeared more vitiated than ever, and the most recent ones had begun to change in colour. It was interesting to know how the same fact struck the two doctors. One remarked, "that with such excretions, there was greater necessity for an alterative than ever"; the other said, "that seeing such had been the effect of the alteratives which the patient had already taken, the sooner they were suspended the better." This led to a warm debate, which was ultimately decided by an appeal to the nurse. Thus: "Nurse, which is the last motion passed?" "This" (the healthy looking one). "How long has it been passed?" "An hour." "What was the appearance of the other motions when they were passed?" "They looked precisely the same as the one first referred to." "Then they have all changed in appearance since they were placed here?" "Yes." "Do the motions change in appearance now more rapidly than they used to do?" "Yes." 'This confirmed the idea that the "vitiated character of the excretions" was, so to speak, a post mortem appearance, and simply indicated a smaller amount of vital force (as opposed to chemical) than is usually possessed by vital products when separated from the body. The termination of the case showed the justice of this view, for the patient's strength continued to diminish, and he died shortly afterwards of pure debility and exhaustion. It is useless to speculate upon what might have been the result had the phenomena been read correctly from the first.

As decomposition takes place in the alvine secretions very rapidly in fevers and all diseases marked by great debility, the practitioner must ever have his attention alive to the fact, that what he calls "vitiation" may be more apparent than real, and a sign which calls for stimulants and strengthening remedies rather than mercurial alterative medicines, whose invariable effect is to make a weak patient weaker.

The excretions of the body being comparatively few, and for other reasons, we cannot prosecute our inquiries into the influence of vitality upon them in the same way as we did concerning the function of secretion. We are not in the habit of requesting our patients to retain their saliva, their tears, their leucorrhceal or catamenial secretions, etc., for our inspection; consequently we know little about the phenomena of decomposition in these fluids and the changes effected thereby.

There are, however, two other excretions with which we are tolerably familiar; namely, the breath and the urine, respecting which we may say a few words. As a general rule, we know that the breath of healthy children is free from any odour perceptible to our senses; and we may say the same of that of healthy adults. But as soon as a child begins to suffer from debility, we know that we can recognise in its breath a variety of unpleasant smells, which we designate as sour, sickly, or putrescent. These odours are increased in intensity and duration by every cause which tends to augment the existing debility; and they go away as soon as the health is restored. In adults, the influence of debility in producing "foul breath" is very remarkable. I know individuals in whom it is invariably produced by a day's fatigue, by the occurrence of the catamenia, or by the too prodigal use of aperient medicines. In these instances, it is cured by wine, tonics, and rest, as certainly as diarrhœa is checked by opium, etc. In other, it comes on in consequence of indulgence in such passions as anger, or from excessive fear, anxiety, and disappointment. In others, it precedes and accompanies asthenic indigestion, and in them a debauch is as surely followed by foul breath, as it is followed in others by nausea and headache. Of course, it may be argued that this foul breath is not due to decomposition of the expired air after it leaves the lungs, but simply to the exhalation of "crudities" already existing in the blood. I acknowledge the force of the argument; but it matters little which solution we accept, so long as we recognise in such phenomena the presence of impaired vital force, and the 462 necessity that exists for adjusting the work to be done to the constitutional power to do it.

There can be no such difficulty respecting the urine. Under ordinary circumstances, this excretion continues for about four and twenty hours, without undergoing any perceptible decom. position; but when the individual passing it is weak, decomposition takes place with a rapidity varying according to the amount of debility present; nay, we may go further, and say that in some instances-as in paraplegia, where the vitality of the kidneys and bladder is very low-the urine is actually decomposed ere it leaves the body. The decomposition is recognised in a variety of ways: by the smell, by the occurrence of vegetable growths, vibriones, torulæ, etc.; by the abundant formation of ammoniacal salts; by turbidity, etc.

It is scarcely necessary to remark that we are fully alive to the fact that atmospheric corditions have a great influence in promoting the rapid decomposition both of urine and frecal matter. What we want to call attention to is, that if on a cool day and in a cool room the urine of a patient shows evidence of decomposition in twelve hours, it is evidence of a debilitated condition of the bladder, the kidney, or the system generally. As such conclusion seems very dull and meagre however, when thus announced, we will endeavour to point it by recording the case which determined the writing of this com. munication.

A medical friend sent me a bottle of urine, with the laconic request that $I$ would give my opinion upon it, and my views of the treatment to be recommended. The sole information he imparted to me was, that it was passed that morning by a boy about ten years old. After allowing the fluid to stand for some hours, it was examined microscopically and by simple chemical tests, etc., but nothing wrong could be found with it, except that it had begun to decompose. The first impulse was simply to communicate that fact to my friend, and leave him to draw his own inference; but as it would be an useful mental exercitation to put down the conclusions which might be drawn from that fact alone, I resolved to give an account of the symptoms that I thought the case would present.

'The following was the train of argument. As there is an absence of much vesical mucus, triple phosphates, etc., there is presumptive evidence against the idea of vesical or rengl disease. There is then general debility; and the presumption is that there will be debility in all the organs of the body, and consequent disorder of function. The signs by which deficient vital power in the brain and nervous system, in the respiratory, digestive, cardiac, and other sets of organs, might generally manifest itself, were then mentioned; and the note ended by recommending a strengthening plan of treatment. In reply to this communication, my friend reported that not only were the symptoms correctly described, but that some had been mentioned in the note which had not been recognised before its receipt. The plan suggested tallied entirely with his own views, and he felt his hands proportionally strengthened thereby.

The practical conclusion to be drawn from the foregoing observations is obvious. If the physician finds that the excretions of any of his patients decompose more rapidly than they would do during health (under the same external circum. stances of light, air, and heat), he may feel certain that the vital powers are seriously impaired; and if under the treatment he adopts the decomposition occurs at an earlier period than it did before, there is reason to inquire whether such phenomenon is due to the augmentation of the disease itself or to the medicines administered for its cure. It will then be a subject for consideration, whether the plan of treatment is to be further developed in the same direction as before, or to be fundamentally changed.

Nor is this a matter of so slight importance as it might at first sight appear. Many seem to think that it is the simplest matter in the world to enable a patient to regain strength; and, consequently, they care little about employing medicines which, while they reduce the powers generally, seem to have some special influence over particular organs. But it is by no means an easy matter to enable a patient to regain health; and any one who systematically endeavours to do so, will have to acknowledge that it is one of the most difficult problems in medicine. It is easy to reduce the strength, for that we have a host of drugs; but directly to increase it, we have literally none.

Sed revocare gradum.........

"Facilis descensus........ 